\title{
Accelerator Experiments and Theoretical Models for the Electron Screening Effect in Metallic Environments
}

\author{
A. Huke, K. Czerski and P. Heide \\ Institut für Atomare Physik und Fachdidaktik, Technische Universität Berlin, Hardenbergstr. 36, \\ 10623 Berlin, Germany

\begin{abstract}
An overview of our experiments and their results concerning the electron screening effects
\end{abstract} \\ in metallic environments are presented. The measurements of the reactions ${ }^{2} \mathrm{H}(\mathrm{d}, \mathrm{p}){ }^{3} \mathrm{H}$ and \\ ${ }^{2} \mathrm{H}(\mathrm{d}, \mathrm{n})^{3} \mathrm{He}$ were performed with an electrostatic accelerator at incident deuteron energies be- \\ tween 5 and $60 \mathrm{keV}$ at different self-implanted target materials. The resulting screening energy \\ values are about one order of magnitude larger compared to gas target experiments and exceed \\ significantly the theoretical predictions. A thorough investigation of the processes in the targets \\ under ion irradiation shows that there are multi-parameter collateral effects which are crucial \\ for the correct interpretation of the observed enhancements. They mainly originate from target \\ surface contaminations due to residual gases in the vacuum as well as from inhomogeneities \\ in the deuteron density distribution in heterogeneous targets. For the special situation of deu- \\ terium in the metallic environment an improved analysis method has been developed beyond \\ the standard procedures. Experimental evidence for the influence of such effects and a math- \\ ematical model for their assessment are given and compared with the results of other groups. \\ We also present a numerical model of the electron screening effect in metallic lattices based \\ on an ab-initio Hartree-Fock simulation.
}

\section{Introduction}

A way for the in depth exploration of the cold fusion phenomena is the reduction of the unknown number of free parameters determining the standard electrolysis and gas cell experiments by the ascription to known and more controlled conditions. Such is done here with accelerator experiments at known energies in a better controllable environment. Therewith, we were first able to demonstrate that the screening energies in deuterated metal targets are one order of magnitude larger than in gaseous targets and hence to provide an initial explanation for the adopted nuclear reaction rate enhancement in cold fusion cells by the screening effect $[1,2,3]$. Meanwhile our results received confirmation from other groups [4, 5, 6, 7, 8, 9]. However, there are particularities and pitfalls in this kind of experiments making them special compared to usual nuclear physics procedures. Taking this not into account results in fatal misinterpretations of the obtained raw data. This is discussed in comparison with results from other groups. The accelerator experiments can furthermore provide access to the branching ratio of the channels of the fusion reactions whose alteration could be observed, too [10]. Additionally to analytic models in [11] a numerical simulation is presented for the pre-reaction impact of the deuterons in the crystal lattice. 


\section{Accelerator experiments}

\subsection{Set-up and data acquisition and analysis}

The experiments have been carried out at an accelerator optimized for low energy beams. Fig. 1 illustrates the principal set-up and the data acquisition system. The accelerator consists of a radio frequency ion source, an acceleration line powered by a highly stabilized $60 \mathrm{kV}$ supply and subsequent electric quadrupoles for focusing and a magnetic dipole for beam analyzation. The beam impinges onto a Faraday cup just inside the target chamber where beam adjustment can be done without disturbing the deuteron density in the targets. A horizontal magnetic steerer is then used to deflect the beam onto the target and removes neutral particles and contaminations carried along by the beam. A cylinder box set to a negative potential surrounds the target in order to suppress secondary electrons. The isolated target holder is connected to a current integrator. The targets were disks made from different pure metals becoming self-implanted deuterium targets under the beam irradiation. Four Si-detectors at the laboratory angles of $90^{\circ}, 110^{\circ}, 130^{\circ}$ and $150^{\circ}$ were used for the detection of all charged particles, $\mathrm{p}, \mathrm{t},{ }^{3} \mathrm{He}$, of the reactions ${ }^{2} \mathrm{H}(\mathrm{d}, \mathrm{p}) \mathrm{t}$ and ${ }^{2} \mathrm{H}(\mathrm{d}, \mathrm{n}){ }^{3} \mathrm{He}$. The detectors needed to be shielded from the backscattered deuterons in order to prevent a congestion of them and the data acquisition system. Therefore grounded Al-foils of thicknesses from $120-150 \mu \mathrm{g} / \mathrm{cm}^{2}$ were placed in front of the detectors. The thickness is sufficient in order to block deuterons up to $60 \mathrm{keV}$ and let pass all other ejectiles. The detector voltage pulses travel through pre-amplifiers and spectroscopic amplifiers. The signals are digitized by four ADC's in an embedded VME system connected to a computer which automatically integrates the proton lines of the spectra in fixed time intervals ${ }^{1}$ and records the four differential counting numbers $N(\theta)$ and the charge $q$ of the integrated beam current at the target in a file which then can be further processed. An example spectrum is shown in fig 1; all ejectile lines are clearly identifiable. Due to the anisotropic angular distribution of the ejectiles of the $\mathrm{d}+\mathrm{d}$ fusion reactions even at the lowest energies, a total counting number $N$ is calculated ${ }^{2}$ providing the tabulated function $N(q)$ which is the basic quantity for the further data analysis.

Correspondingly, the yield from the experiment is given by

$$
Y(E)=\frac{z e}{\varepsilon} \frac{d N}{d q}
$$

where the number of impacting projectiles is already substituted by their charge, $\varepsilon$ is the detector efficiency and $z$ the charge state of the projectile. On the other hand the yield is calculated for an infinitely thick target (regarding the projectile range $R$ ) from scattering theory by

$$
Y_{\text {theo }}(E)=\int_{0}^{R} n \cdot \sigma(E(x)) d x
$$

with the number density of the target nuclei $n$ and the cross-section $\sigma$. Unlike other chemical compounds the small hydrogen atoms are not trapped in firm chemical bonds with metals. The hydrogen density is not bound to a fixed stoichiometric ratio and can and indeed does change under ion irradiation. Changes in the yield may now originate from both the density and the cross-section and need to be discriminated. The density is here a function of the depth, the projectile energy, the implanted charge, the beam flux and other material dependent and environmental conditions. The tabulated function $N(q)$ provided by our data acquisition system makes it possible to retain the differentiation in (1) and thereby gain information on the charge development of a depth averaged density $n(q)$. So assuming depth homogeneity of the deuteron density in (2) the depth $x$ can be substituted by the projectile energy $E$ with the stopping power differential equation [12]

$$
\frac{d E}{d x}=-\left(c_{M}+\frac{n(q)}{n_{D}} c_{D}\right) \sqrt{E}
$$

\footnotetext{
${ }^{1}$ down to $10 \mathrm{~s}$ limited by the serial line

${ }^{2}$ see also [10]
} 


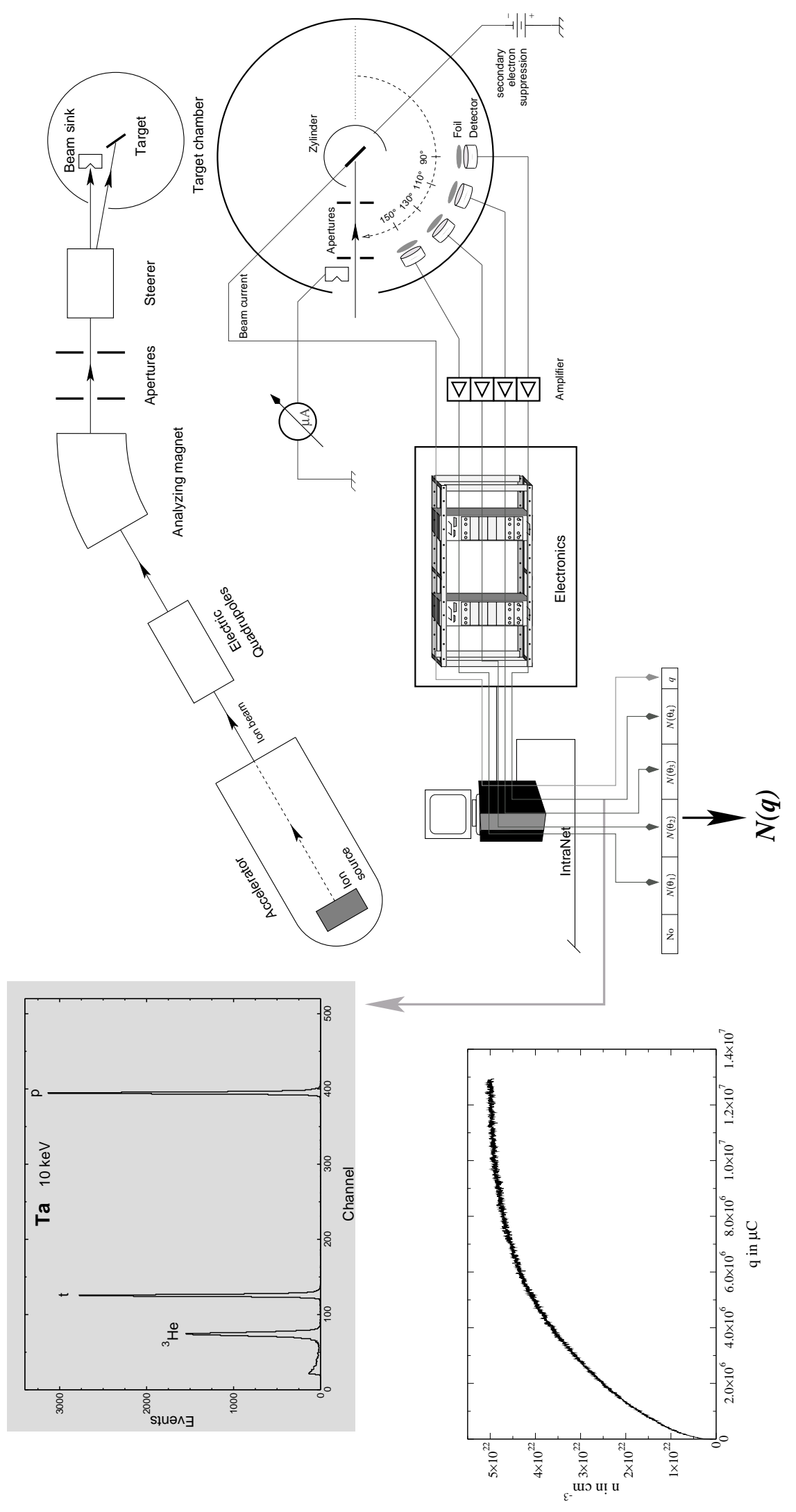

Figure 1: Experimental set-up 
where $c_{M}$ and $c_{D}$ are the stopping power coefficients in the metal and in hydrogen, $n_{D}$ the appendant hydrogen density. One arrives with this substitution at a motivation and an interpretative expression for the here defined reduced yield

$$
y(E ; q):=\frac{Y(E ; q)}{\int_{0}^{E} \frac{\sigma(E)}{\sqrt{E}} d E}=\frac{n(q)}{c_{M}+\frac{n(q)}{n_{D}} c_{D}} \times F(E) .
$$

Since both the cross section in the metallic environment and the deuteron density are unknown the yield need to be set in relation to a known gas target cross section. We therefore chose the parameterization from [13] because they have the highest precision. It forms together with the low energy function $(\sqrt{E})$ of the stopping power (3) the integral in the denominator on the right hand side. The gray printed expression is per se a constant. So if the reduced yield is not constant it is based on deviations of the prescribed progression in the cross-section or the functional dependency of the stopping powers or changes in the density. It is a sensitive measure for such deviations but the distinction of the possible reasons is a matter of reasonable interpretation. Fig. 2 shows plots of the reduced yield at two different energies. One can see long term

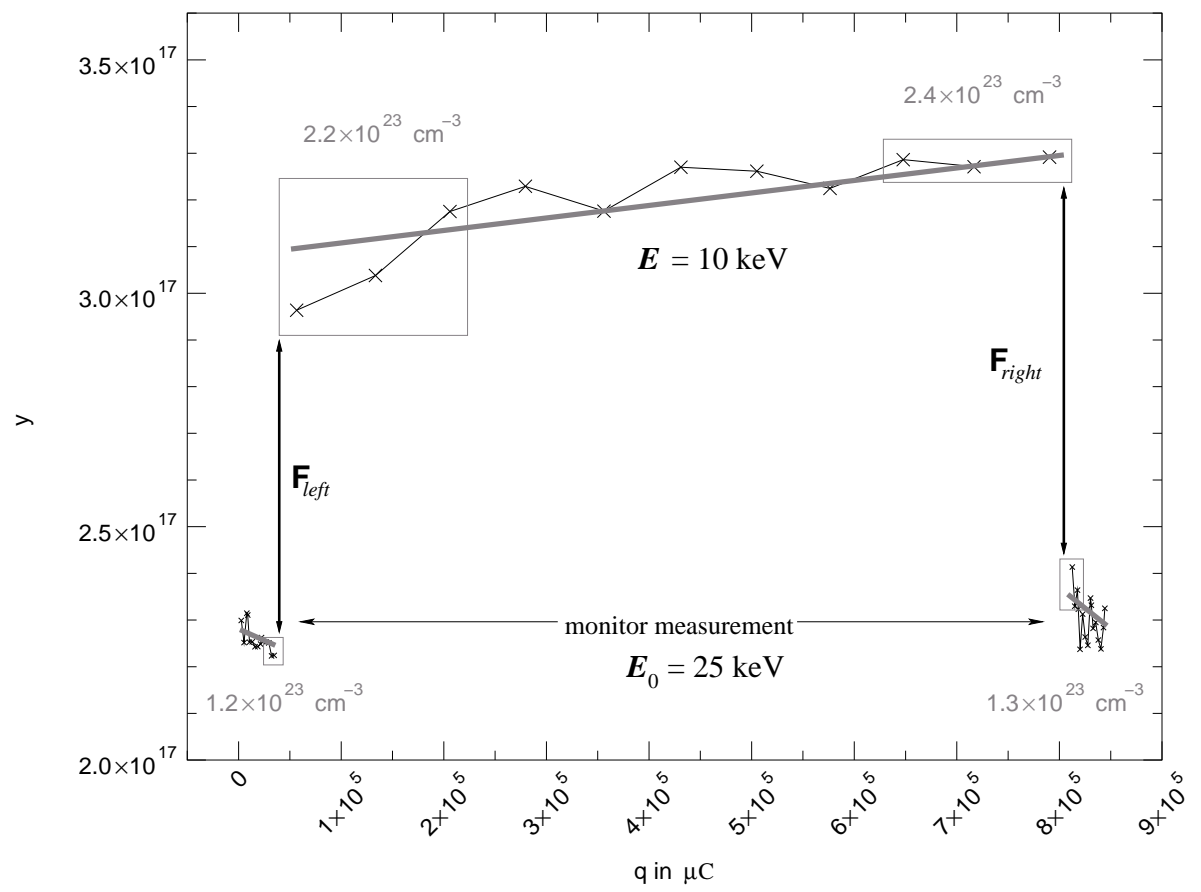

Figure 2: Analysis procedure at the example of Zirconium at $10 \mathrm{keV}$

changes in the individual measurements indicated by the straight lines. These are attributed to changes in the density profiles scattered by the counting statistics, of course. In contrast, the large discontinuities of the reduced yield at the switching of the beam energy result from a modification of the cross section. This is taken into account by the enhancement factor $F(E)$ in (4). Since the absolute quantity of the deuteron density is unknown for the practical analysis a normalized enhancement factor is defined

$$
F_{\text {norm }}(E):=\frac{y(E)}{y\left(E_{0}\right)}=\frac{F(E)}{F\left(E_{0}\right)}
$$

with the normalization energy $E_{0}$ which is chosen to be $25 \mathrm{keV}$ for the monitor measurements. The gray rectangles indicate the points from which the error for $F_{\text {norm }}$ is inferred. Thus not only errors from the counting statistics but also from long term changes of the density are included. Results are displayed in fig. 3. Assuming electron screening as the reason for the enhancement and adopting $U_{e}$ as a kinetic energy 


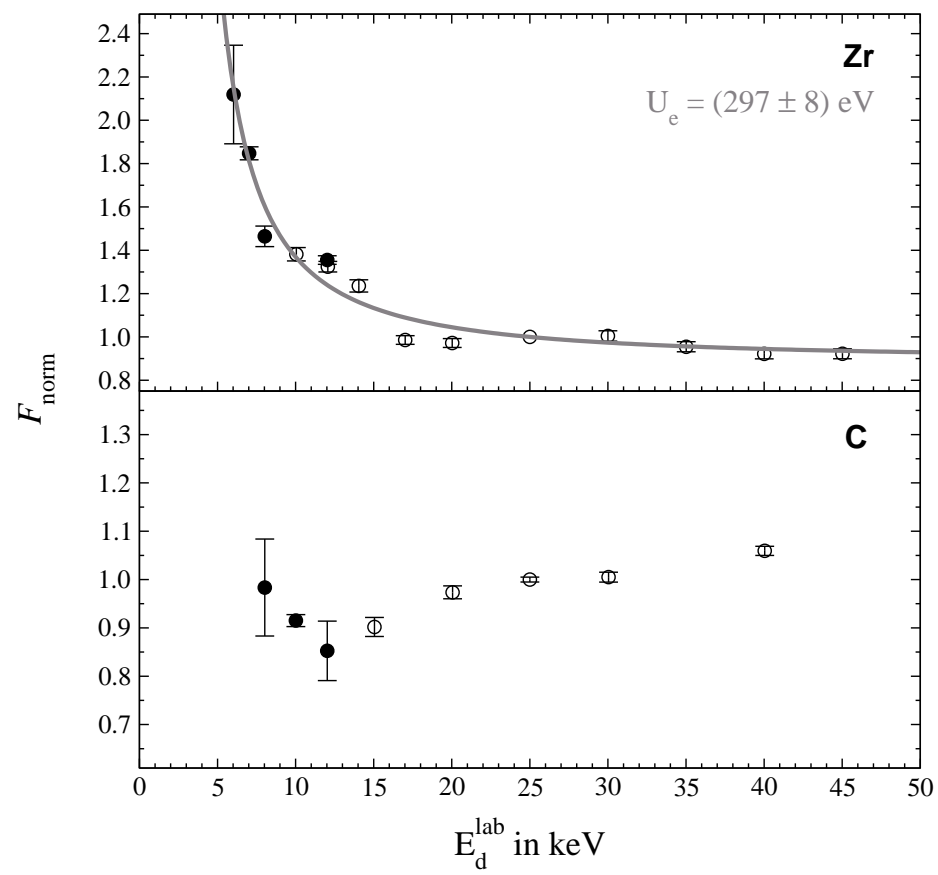

Figure 3: Exemplary results for the enhancement factor $F_{\text {norm. }}$ Screening enhancement for $\mathrm{Zr}$ theoretically described by the curve with the single parameter $U_{e}$. No enhancement for carbon.

shift parameter in the cross-section [14] of the yield one receives

$$
F(E)=\frac{\int_{0}^{E} \frac{\sigma\left(E+2 U_{e}\right)}{\sqrt{E}} d E}{\int_{0}^{E} \frac{\sigma(E)}{\sqrt{E}} d E}
$$

for the screening enhancement factor of thick target yields ${ }^{3}$. The corresponding curve in fig. 3 fits well to the data supporting the screening hypothesis. Our data analysis procedure is thus independent of the absolute value of the deuteron densities inside the targets and the stopping power coefficients with errors from $10-20 \%$. The functional dependency of the stopping powers on the energy $\sqrt{E}$ has been repeatedly confirmed, see [16] and references therein. The reduced yield can be used to calculate a deuteron density estimate by solving (4) towards $n(q)$ and supposing $F=1$. Only for this purpose the stopping power coefficients are explicitely required. A corresponding density plot for an initial implantation in $\mathrm{Al}$ is shown in fig. 1. The numbers above the gray boxes in fig. 2 are density estimates for that areas.

\subsection{Experimental specialties and pitfalls}

The investigation of nuclear reaction cross-sections on deuterium in metals should be performed at the lowest possible energies. This means that the composition of the topmost atomic layers of the metallic target is of crucial importance because of the quickly decreasing range of the beam ions, considerably below $1 \mu \mathrm{m}$. This exactly is unusual for experimental nuclear physics. The usual set-ups in experimental nuclear physics are constructed in high vacuum technology. But here the contained water vapour from the surfaces

\footnotetext{
${ }^{3}$ The screening energy $U_{e}$ should only be applied to the Coulomb barrier penetration in $\sigma$, see $[11,15]$. The correction becomes only important for far lower beam energies.
} 
of all materials leads under ion impact to a progressing oxidation of the metal because of the stronger electron negativity of oxygen in comparison to hydrogen. Hence hydrogen is contained in metal oxides only in segregation at low and unstable densities. Consequently the oxidation diminishes and eventually destroys the screening effect with the growth of the metal oxide layer. Carbon hydrides contained in HV systems pose another problem leading to carbon layers on the target as will be discussed below. In such a way generated alterations in the depth profile of the deuteron density distribution in the target is the singular dominating error source for the observed enhancement and the inferred screening energies. Our vacuum system is made of aluminium with elastomer gaskets pumped by turbo molecular pumps with auxiliary oil lubricated two stage rotary vane pumps and $\mathrm{LN}_{2}$ cooled cryogenic traps. A residual gas analyzer (RGA) was used in order to monitor the composition of the residual gas in the vacuum. In accordance to the literature about HV systems the main constituent of the residual gas is water. Water vapour is due to its extraordinarily high dipole moment very adhesive to solids and is hence chemisorbed to surfaces. Now under the ion irradiation several processes are enabled. Via heating and phonon excitation at the surface the beam provides the activation energy for dissociative chemisorption of the water molecule, i.e. the protons are splitted off and the remaining oxygen radical forms a chemical bond to the metal atoms. Essentially the same happens by direct impact excitation of the water molecule by the ions. The hydrogen implantation into the metal causes aside from the usual surface deterioration a in depth destruction of the crystal integrity of the material known as embrittlement which always occurs if the hydrogen loading rate is too high and not proceeding in thermal equilibrium [10,17]. Thus the surface is fractalized and the oxidation can progress into the bulk of the metal quickly creating a thick metal oxide layer. Fig. 4 contains as an example for it a picture of the surface of an $\mathrm{Al}$ target which turned into a sponge like structure. The rate of the oxidation

Al

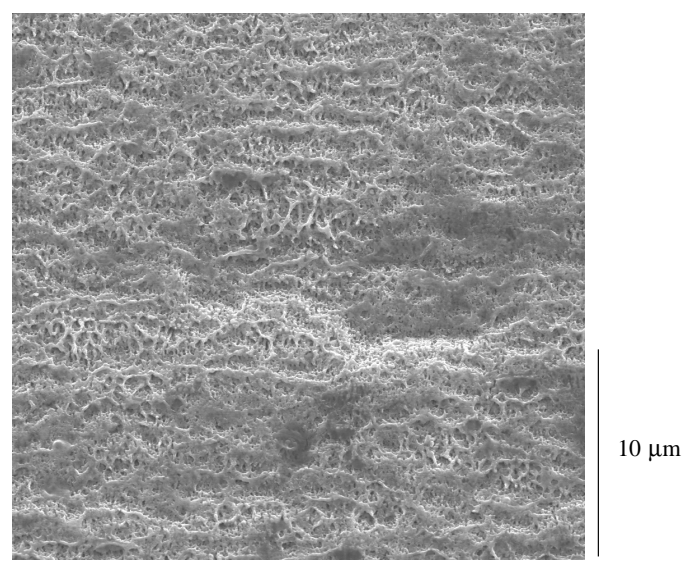

$\mathrm{Ta}$

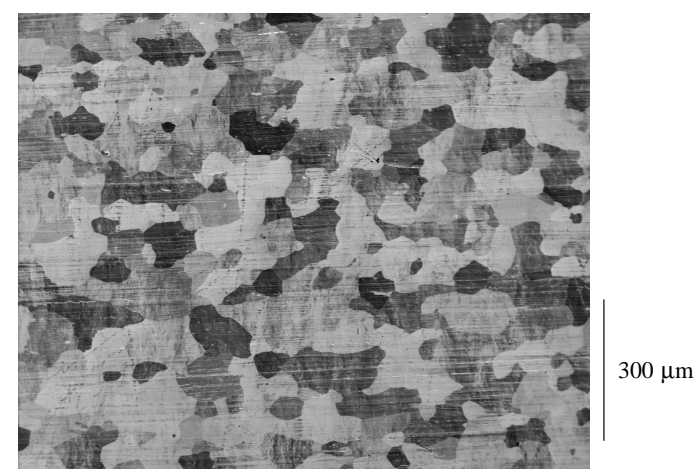

Figure 4: Scanning electron microscopic pictures of target surfaces. Left: Symptoms of embrittlement for Al. Right: Beginning layer formation for Ta in island growth mode.

process depends on the concrete form of the mutual interaction potential between the water molecule and the surface atoms, establishing a material dependency. The energy supply of the beam enables these processes even for the noble metals. Albeit generally spoken, more reactive metals apt more to oxidation and embrittlement while for the latter the structural difference between the metal and the metal hydride is more important. Aside from the overall beam heating the energy of the projectiles is also important because lower energy projectiles are more effective at the surface [18]. The partial pressure of water in $\mathrm{HV}$ is so high that there are ample supplies for the surface reactions. The hit rate of water molecules with a sticking coefficient of almost one is in comparable orders of magnitude as usual beam currents of $10-100 \mu \mathrm{A}$. This implies a dependency on the ion flux, too. There are two counteracting processes: Sputtering and thermal or ion stimulated desorption. The sputtering yield of the lightweight deuterons is far too low in order to keep the surface clean with the resulting sputtering rate. One would expect that an increased temperature of the surface would increase the desorption rate of the water molecules. If the activation energy barrier for dissociative chemisorption of water is positive an increased temperature 
yet proliferates the oxidation ${ }^{4}$. Similar is valid for ion stimulated desorption/chemisorption. Such again depends on the interaction potential but usually oxidation prevails. Unless UHV systems equipped for entire baking are used the oxidation cannot be avoided. A deuteron irradiation of only $1 \mathrm{C}$ is enough to produce a considerable metal oxide layer, see [20, fig. 1]. There is, however, a process that is nonetheless able to prevent oxidation: large carbon hydride molecules, e.g. backstreaming from the forepumps, can be physisorbed at the surface, cracked up and the carbon atoms can react with the oxygen radicals to carbon monoxide keeping in that way the surface clean. Differently from water, carbon hydrides are physisorbed to surfaces. The strength of this weaker bond increases with growing molecular mass. The ratio of absorption and desorption under the ion irradiation has similar dependencies. An evidence for this chemical surface reaction is the detection of a considerable $\mathrm{CO}$ fraction by the RGA which was below the detection threshold without beam irradiation. These processes were thoroughly explored by the regulated infusion of decane with monitoring feedback. The surface can only be kept clean if the fraction of water and carbon hydrides in the residual gas are in an equilibrium which is of course also dependent on prementioned parameters. If the fraction of carbon hydrides is too low the surface will oxidize. If it is too high a carbon layer will build up. Both is essentially irreversible. Fig. 5 shows some of the results of these experiments for Ta demonstrating the high spread in the inferred screening energies depending on the surface composition which were verified by $\mathrm{EDX}^{5}$. In order to limit the layer formation the totally implanted charge was reduced. For the analysis

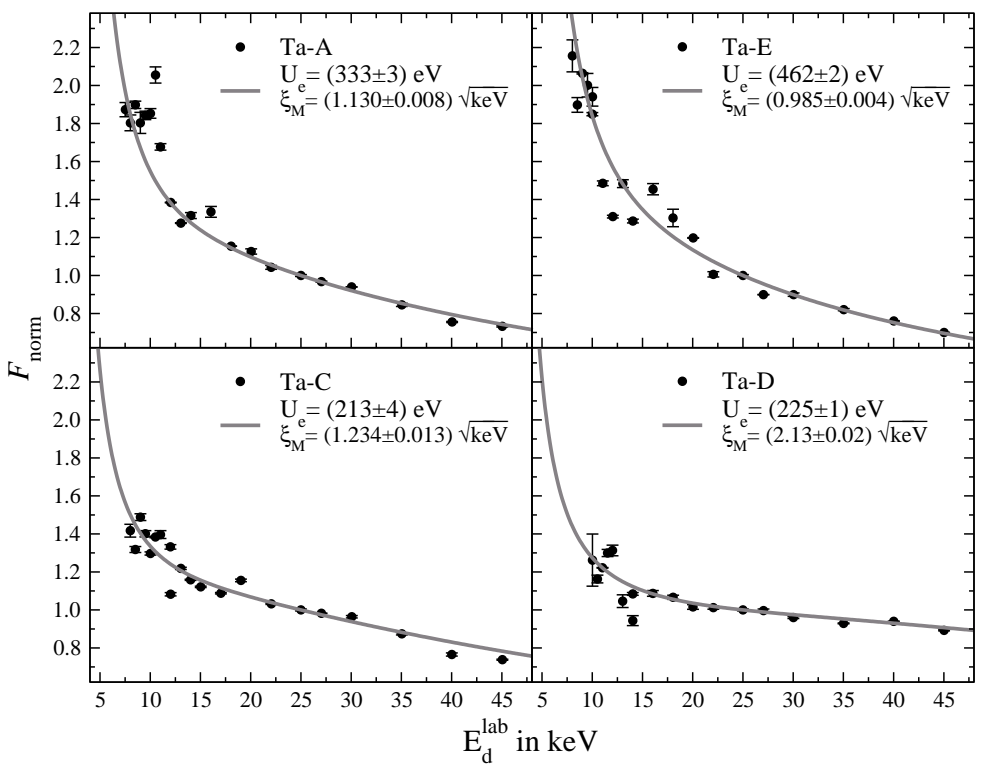

Figure 5: Effects of different surface compositions on the inferred screening energy for Ta. Ta-A has a small C-excess, Ta-E has slight C-traces, Ta-C a thick C-layer, Ta-D a thick $\mathrm{MO}_{x}$-layer.

a more sophisticated expression for the yield in $(4,5)$ was used based on a model of the target with three stacked layers [20]: The top layer consisting of either metal oxide or carbon, a deuterized zone of the metal and the bulk of the metal containing essentially no hydrogen. Each can have different thicknesses and relative deuterium contents. The results for $U_{e}$ in fig.5 were obtained with only the additional parameter $\xi_{M}$ for the thickness of the deuterated zone in the metal in energy equivalent units of the stopping. The differences for Ta-A and Ta-E are already considerable though the thicknesses of the surface layers were small and just started forming. Fig. 4 shows the beginning of the formation of a carbon layer starting from islands which will eventually cover the whole surface in concordance with experiences from thin film technology [18]. Ta-C has already a relatively thick carbon layer which strongly reduced the screening energy. Just as the metal oxide layer does in Ta-D. Those layers were just thick enough in order to be included in the model and infer their thickness. The thickness of the metal oxide layer is $0.09 \sqrt{\mathrm{keV}}$, which

\footnotetext{
${ }^{4}$ see e.g. [19] or any surface physics textbook

${ }^{5}$ Electron dispersive X-ray micro analysis.
} 
conforms to about $7 \mathrm{~nm}$. The corresponding screening energy would be $433 \mathrm{eV}$. $15 \mathrm{~nm}$ are enough to let the screening enhancement completely vanish. Much thinner surface layers already reduce the inferred screening energy considerably. So the real value for the screening energy of Ta is possibly around $400 \mathrm{eV}$. Carbon can achieve high densities but it does not show the electron screening effect as fig. 3 proves. Thin deuterated carbon layers can, however, simulate a screening enhancement as inhomogeneous density profiles can do [20]. As already said, the metal oxide contains only few deuterium in segregation. Those low densities are unstable and change under different conditions. At the example of a Na target with a very thick metal oxide layer the development of the density is illustrated in fig. 6 . The density estimates are
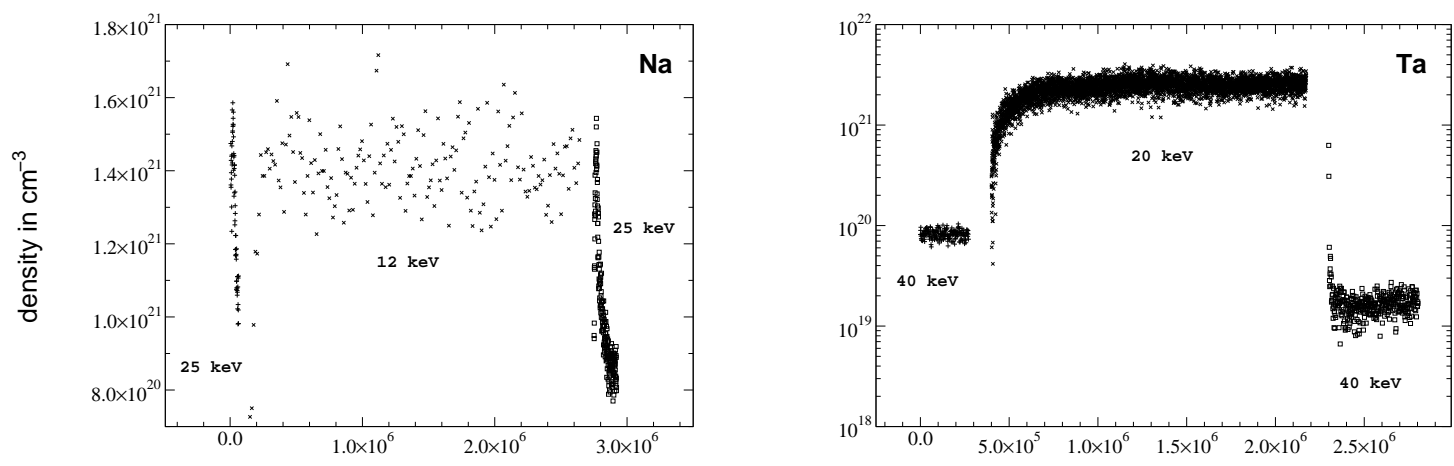

charge in $\mu \mathrm{C}$

Figure 6: Development of low deuteron densities. Left: Na target with a thick metal oxide layer. Right: A beam heated thin Ta foil.

calculated from the reduced yield as previously described. Before the monitor measurement at $25 \mathrm{keV}$ a measurement at a low energy had been taken. The density quickly decreased then at $25 \mathrm{keV}$. Thereafter a measurement at $12 \mathrm{keV}$ were started. The density very quickly increased reaching a higher level than at $25 \mathrm{keV}$. But the discontinuity at the beginning was in the wrong direction. The density for the sequencing monitor measurement started once again at a high density which quickly decreased. The discontinuity at the beginning was once again in the wrong direction. So there is definitely no screening. The quick shifts in the densities after the change of the implantation energy going to a 'saturation' level originate from a shift of the deuteron distribution depth profile in the metal oxide linked to the different ranges of the ions. With our method of recording a yield function $Y(q)$ over the implanted charge we can recognize those shifts and reject them. If, however, only the total yields of the long time measurements are regarded as in the standard method their comparison would erroneously lead to a screening interpretation. The same problem arises when working with low implantation densities below the stoichiometric ratio even when the metal oxide layer is negligible. Except for insufficient implantation the density remains low if the thermal energy of the deuterons is higher than their chemical binding energy to the metal so that they can float. This applies mainly to transition metals with low ability to bind hydrogen (groups 6A-8A, 1B) or if the metals are heated. An example for the consequences of heating is shown in fig. 6 for a Ta-foil of $7 \mu \mathrm{m}$ which was heated by the beam power. One observes the same behaviour and no real screening enhancement. The density returns to an equal saturation level if the surrounding conditions are the same, i.e. same beam energy, current, target heat flow etc. The most effective heat transportation mechanism in solids is the free electron gas. Cooling the target holder has little effect since the thermal resistance at the connection is very high. Besides from heating the density profile of the deuterons in target materials with low binding ability for deuterons (metal oxides, metals with low affinity to hydrogen, metals at high temperatures) is also changed by direct projectile hits and close phonon generation at the target deuterons depending on the beam energy. Furthermore, the metal oxide as a thermal insulator will be considerably heated by the beam power. It is therefore preferable to use thick target disks at moderate temperatures with high densities. On the other side, cooling a target to very deep temperatures would transform it into a cryogenic trap accumulating water in thick layers on its surface prior to irradiation promoting the oxidation. The detailed investigation is covered in [3]. 


\subsection{Comparison}

Paying attention to all the above discussed experimental problems we can state that our results represent lower limits to the real screening energy values: $(190 \pm 15) \mathrm{eV}$ for $\mathrm{Al},(297 \pm 8) \mathrm{eV}$ for $\mathrm{Zr},(313 \pm 2) \mathrm{eV}$ for Pd and $(322 \pm 15) \mathrm{eV}$ for Ta. Due to our monitoring method we estimate the upper limit of $U_{e}$ to be probably not larger than additional $100 \mathrm{eV}$. The value for $\mathrm{Sr}$ ranges between 350 and $800 \mathrm{eV}$ since the measurement was impaired by layer formation, even more for Li where only an upper bound of $150 \mathrm{eV}$ could be determined and no screening for $\mathrm{Na}$ (fig. 6). Two tests with $\mathrm{Y}$ and $\mathrm{Er}$ led to thick metal oxide layers, too. In fig. 7 an overview of screening results from other experiments is plotted. Historically the

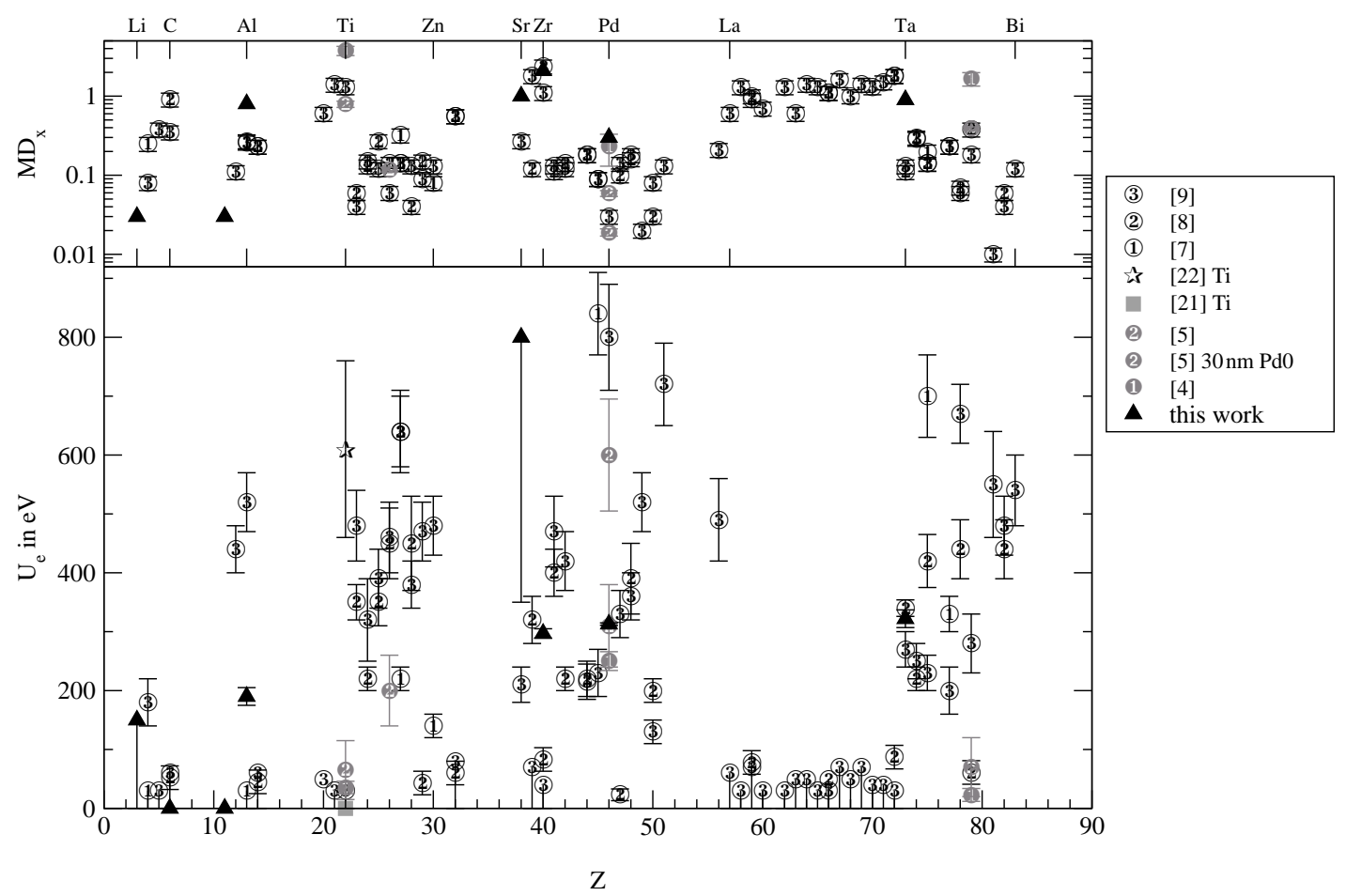

Figure 7: Overview of screening experiment results. Bottom: Screening energies $U_{e}$. Top: Deuterium to metal ratio $x$. The values for $x$ of [5] were estimated from fig. 2 therein. The values of [9] are the data base; data points from $[8,7]$ are included if they differ, only.

two elements Pd and Ti were of special interest. So one of the first accelerator experiments was done on Ti [21]. The authors made no effort to determine the deuteron density but used a literature value and obtained no enhancement. All further measurements on Ti resulted in very low screening values. The higher the deuteron density, the lower the screening value. Ti is chemically very similar to $\mathrm{Zr}$, both belong to the group 4A. From our experience $\mathrm{Zr}$ oxidizes readily. So a relatively thick metal oxide layer explains the results and the discrepancy to the value in [22]. It was obtained with a glow discharge of $\sim 0.5$ A deuteron current. Despite of the low sputtering yield of the deuterons the high current permits a sputtering rate which is so high that it can impair the oxidation enough. A deliberately produced $30 \mathrm{~nm}$ thick PdO layer on a Pd target in [5] yielded an especially high screening energy with a low density obtained from the total yield only. Such a thick PdO layer would show quick shifts in the density profiles with higher averaged densities at lower projectile energies like in the Na example in fig. 6 when changing the projectile energy and using the differential analysis method. So this screening is simulated by the density alteration in the total yield. The same applies to the extraordinary high screening value for Pd of [9] at a low density. The screening energies for Pd of [4] and [5] agree within their errors though obtained at very different densities and both at deep temperatures. The value in [5] is in concordance with our result. For Au there is a discrepancy between [4] and [5] in the densities at low screening energy values and deep temperatures. 
We made a test with a thin Au foil and observed a behaviour like for the Ta foil in fig. 6 without the screening enhancement discontinuities. While the targets as described in [5] are thick enough $\sim 1 \mathrm{~mm}$ to guarantee an effective heat transport in the bulk of the material by the electron gas, the heterogeneous target $\mathrm{Au} / \mathrm{Pd} / \mathrm{PdO}$ with a total thickness of only $60 \mu \mathrm{m}$ (thereof $0.1 \mu \mathrm{m} \mathrm{Au}$ ) is too thin therefore leading to a considerable temperature increase in the beam stopping volume which is to this extend not detectable by an outside mounted thermocouple. So the observed high screening energy of $(602 \pm 23) \mathrm{eV}$ can be explained by the shifts in the density profile due to elevated temperatures and the heterogeneity of the target and accordingly the density. In order to explain the relation between the screening energy and the density the concept of a deuteron 'fluidity' was introduced in [5] where fluid deuterons and conduction electrons are to behave like a hot plasma. But in palladium oxide there are no conduction electrons. In view of the stated density dynamics this explanation is decrepit. The explanation by density dynamics is also sustained by the significantly larger standard deviations of the repeated density measurements at $10 \mathrm{keV}$ for targets with low densities in [5, fig. 2]. Indeed the saturation density in our experiments returns to the same level for the same conditions but with higher deviations. The largest data set of screening energies is provided by $[9,8,7]$. They only observed the total yield of the measurement, too. The density is determined by a global fit to the previously extracted relative cross-sections by using the unprecise stopping power coefficients to a known cross-section at $30 \mathrm{keV}$ and consequently obtains a density estimate which is valid at $30 \mathrm{keV}$ only [6]. The intention is to find a connection between the observed screening energy and some electronic properties of the elements. The authors propose the Hall coefficient to be this quantity stating that the free charge carriers, i.e. electrons and holes likewise, form a Debye sphere around the deuterons and thus generate the screening potential. The classical Debye screening is, however, not applicable for low temperatures (electron energies below the Fermi energy) and dense plasmas (solid states) where the quantum mechanical effects dominate and the screening effect depends only on the charged particle density and not on the temperature [23]. Additionally the motion of the bound electrons simulating the hole is not free but governed by quantum mechanical tunneling between neighbor atoms. The fact that the screening energy is vanishing for high deuteron densities is explained by the assertion that these metal hydrides are insulators. This is not right for the majority of the metal hydrides which are metallicly or covalently bound and retain their metallic properties. The Baranowsky-curve of the electric resistance of metal hydrides shows that the resistance at the chemical stoichiometric ratio is even lower than for somewhat lower densities and comparable to the metal. Using a ${ }^{3} \mathrm{He}$ beam on a deuterated ${ }_{78} \mathrm{Pt}$ target via the reaction $d\left({ }^{3} \mathrm{He}, \mathrm{p}\right){ }^{4} \mathrm{He}$ a screening energy was inferred about twice as high as for the $\mathrm{d}$ beam which was regarded as a confirmation of the $Z$ dependency of the Debye hypothesis [8]. In [9] however the screening energies for ${ }^{3} \mathrm{He}$ and $\mathrm{d}$ beams at $\mathrm{Pt}$ became equal. The homogeneity of the depth distribution of the deuterons in the targets was reconfirmed by a subsequent off-line ERDA ${ }^{6}$ with a $4 \mathrm{MV}$ tandem accelerator [7]. Pointed to the problem of oxidation RBS ${ }^{7}$ analysis was performed on the targets with the result that there were 'no detectable surface contaminations' with the exception of $\mathrm{Al}$ where there was an $\mathrm{Al}_{2} \mathrm{O}_{3}$ layer with a thickness of about 150 monolayers. Then a $\mathrm{Kr}$ ion sputtering treatment at 15 or $35 \mathrm{keV}$ was applied prior to the implantation measurements in order to remove natural metal oxide layers which is the main difference from [7] to [8] and [9]. This procedure does not take into account that the major cause of the oxidation is contributed by the water in HV systems under deuteron irradiation. For both ERDA and RBS it is valid that light projectile ions with a kinetic energy of some MeV cannot provide a wide energy spectrum of the ejectiles which would be necessary in order to resolve single atomic layers. Therefore a HIERDA ${ }^{8}$ with incident energies of the heavy ions in the $0.1 \mathrm{GeV}$ order of magnitude would be required with sophisticated magnetic analyzing systems. This is additionally complicated by the circumstance that these methods deliver expressive results only if heterogeneous samples are made up of well defined layers. This is not fulfilled for the implantation targets with indistinct chemical composition and surfaces fractalized by embrittlement and beam deterioration. So the applied methods are not able to detect metal oxides with a thickness of a few tens monolayers (some nanometers) which is already sufficient to obliterate the screening enhancement while they are not thick enough to affect the density determination at $30 \mathrm{keV}$ significantly. While the high sputter yield of the $\mathrm{Kr}$ ions may allow for a surface

\footnotetext{
${ }^{6}$ Elastic Recoil Detection Analysis

${ }^{7}$ Rutherford Back Scattering

${ }^{8}$ Heavy Ion Elastic Recoil Detection Analysis
} 
cleaning the large $\mathrm{Kr}$ atoms thoroughly destroy the crystal structure of the target and get trapped in the material fractalizing the surface and thus possibly even promoting the oxidation process under subsequent deuteron irradiation since the necessary annealing is omitted. The deviations in the screening energies between [7], [8] and [9] are in both directions, anyhow giving an indication for the magnitude of the true error in the determination of the screening energies in this way similar to our dedicated experiments on Ta (fig. 5). Like in the data of $[4,5]$ there is a clear connection between the density and the screening energy. High densities are linked to low screening energies because of moderately thick metal oxide layers. Examples are the elements of the groups $3 \mathrm{~A}\left({ }_{21} \mathrm{Sc},{ }_{39} \mathrm{Y}\right.$ and the lanthanoides $\left.Z=57-71\right)$ and $4 \mathrm{~A}(\mathrm{Ti}, \mathrm{Zr}$ and ${ }_{72} \mathrm{Hf}$ ) emphasizing the chemical kinship with regard to the described surface reactions. Low densities generate high screening energy findings due to shifts in the density profile either in thick metal oxide layers or materials with low hydrogen binding ability. Such can be recognized at the transition metals (groups 6A-8A: $Z=24-28,42-46,74-78$ ) for example. In contradistinction thereto our high screening energy results were obtained at high densities close to the chemical stoichiometric ratios. Using the data of $[9$, tab. 1] a Spearman rank correlation calculation can be done. For the test of a correlation between $U_{e}$ and the deuterium ratio $x$ one obtains $r_{S}=-0.800$ and $P=5.1 \cdot 10^{-14}$, which means a rather high correlation with a very high significance. On the other side, the result for a correlation test between $U_{e}$ and the effective charge carrier concentration $n_{\text {eff }}$ calculated from the Hall coefficient is $r_{S}=0.489$ and $P=0.013$, which is a medial correlation with a weak significance. This is not enough in order to rule out the testing null hypothesis of no correlation thus deprecating the Debye hypothesis, too.

\section{Numerical Simulation}

In $[11,15]$ we presented a analytical model based on the dielectric function theory for the description of the screening effect and extrapolation to room temperatures. It remains, however, below the measured screening energies by a factor of 2 though better than other approaches cited therein. Therefore a first effort was undertaken to simulate the pre-reaction impact with an ab-initio quantum mechanical HartreeFock calculation which is able to consider the actual crystal structure while the analytical model only operates on averaged material properties. The concrete form of the electron density distribution around the nucleus indeed influences the screening energy to a great extend as was shown in [24] on a $\mathrm{D}_{2}$ molecule with a time dependent Hartree-Fock calculation. Since only workstation class computational power was available several too farreaching simplifications needed to be implemented. The motion of the projectile needed to be abandoned for an adiabatic limit. Only a very small LiD crystal consisting of $6 \mathrm{Li}$ atoms on a limited set of basis functions were feasible for the calculation which lasted 1.6a netto. The calculations were done with the quantum chemical program GAUSSIAN [25]. An intersection of the electron probability densities is plotted in fig. 8. It is actually the difference between the molecular density and that of the single atoms thus exposing electron transfers at the chemical bond. One can clearly see an increase of the electron density at the deuterium on the expense of the $\mathrm{Li}$ atoms in concordance with the ionic nature of this bond. This is even enlarged during the approach of the colliding deuterons. The electronic force between the two deuterons is plotted in fig. 8. Its integration yields the screening energy which is with $43.4 \mathrm{eV}$ twice as high as from the simple model [14] but still far below the measured values. Using enhanced basis sets leads to an increase of the calculated screening energy. A complete treatise can be found in [3].

\section{Conclusion}

We developed a differential data analysis method which gains the maximum information from the raw data. The method is independent of the unprecise stopping power coefficients and the actual absolute value of the deuteron number density in the targets. It allows for the recognition and rejection of measurements with unwanted shifts in the density depth distribution profile thus preventing the erroneous extraction of an artificial screening enhancement in contrast to the standard analysis based on the total yield measurement used by $[4,5,7,8,9]$. Those undesirable density profile changes occur in targets with low hydrogen 

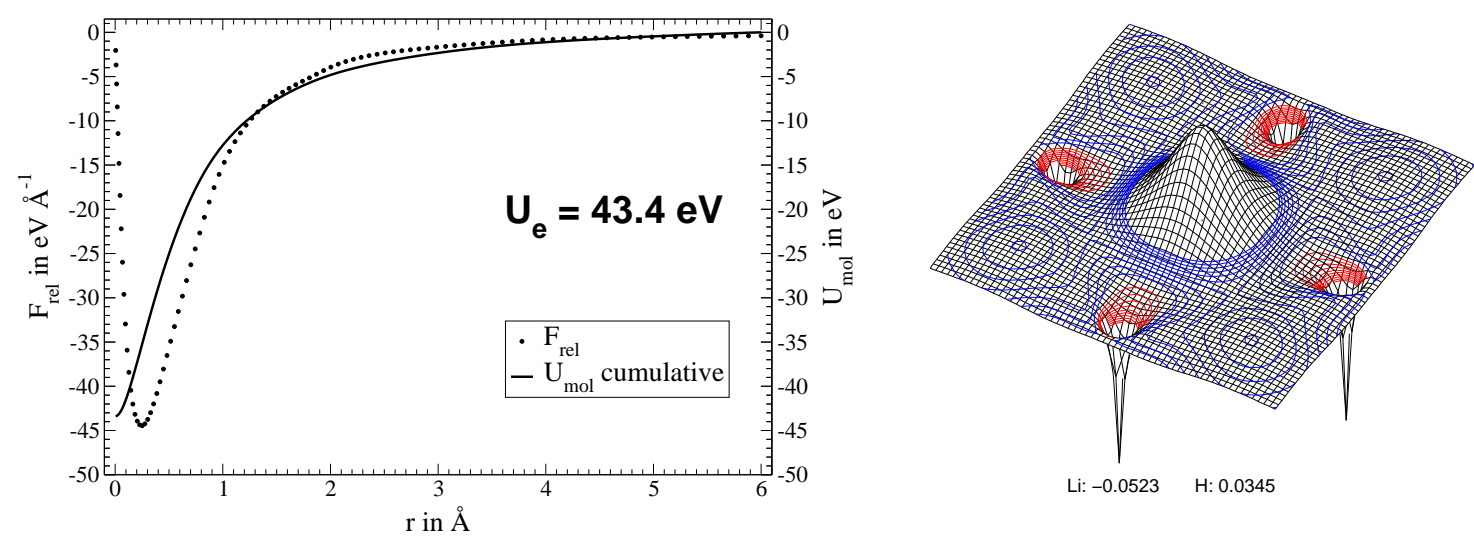

Figure 8: Left:Screening force and cumulatively integrated potential during the pre-reaction impact. Right: Electron probability density on a plane with $4 \mathrm{Li}$ atoms at the sites and the target deuteron at the center.

binding ability, like many of the transition metals, at elevated temperatures and heterogeneous targets with metal oxide or carbon layers or different (relatively) thin metal layers. The fatal alteration of the inferred screening energies due to layer formation under beam irradiation depends on many parameters and is inevitable in high vacuum systems that are used by all groups. The deviation from the 'real' value of the screening energy is probably around $100 \mathrm{eV}$. So any conclusion based on the observed material dependence of the screening energies is premature. On the other hand, the theoretical calculations performed within an improved dielectric function theory $[11,15]$ predict only a weak material dependence of $U_{e}$ in contradiction to the experimental results of $[7,8,9]$. Furthermore, an error of $100 \mathrm{eV}$ implies an error in the reaction rate of many orders of magnitude when extrapolating to room temperature, i.e. the cold fusion condition $[11,15]$. Consequently, aside from the sustained fact that there is a great screening enhancement at this time no further assertion can be made. For a precise determination of the screening energies ultra high vacuum systems with pressures well below $10^{-10} \mathrm{hPa}$, where only hydrogen and noble gases are in the residual gas, and equipped with in-situ target diagnosis techniques are mandatory.

The presented numerical simulation bases on a too simplified model due to limited computational power hence failing to reproduce the observed screening energies while already exposing shifts in the electron distribution. An extended model capable of describing the situation realistically would require massive parallel supercomputers though very instructive insights into the electron dynamics for the understanding of the mechanism can be expected.

\section{References}

[1] K. Czerski, A. Huke, P. Heide, M. Hoeft, and G. Ruprecht. In N. Prantzos and S. Harissopulos, editors, Nuclei in the Cosmos V, Proceedings of the International Symposium on Nuclear Astrophysics, page 152, Volos, Greece, July 6-11 1998. Editions Frontières.

[2] K. Czerski, A. Huke, A. Biller, P. Heide, M. Hoeft, and G. Ruprecht. Europhys. Lett., 54(4):449-455, 2001.

[3] A. Huke. Die Deuteronen-Fusionsreaktionen in Metallen. PhD thesis, Technische Universität Berlin, 2002. http://edocs.tu-berlin.de/diss/2002/huke_armin.htm.

[4] H. Yuki, J. Kasagi, A. G. Lipson, T. Ohtsuki, T. Baba, T. Noda, B. F. Lyakhov, and N. Asami. JETP Lett., 68(11):823, 1998.

[5] J. Kasagi, H. Yuki, T. Baba, T. Noda, T. Ohtsuki, and A. G. Lipson. J. Phys. Soc. Jpn., 71:2281, 2002. 
[6] F. Raiola et al. Eur. Phys. J. A, 13:377, 2002.

[7] F. Raiola et al. Phys. Lett. B, 547:193, 2002.

[8] C. Bonomo et al. Nucl. Phys. A, 719:37c, 2003.

[9] F. Raiola et al. Eur. Phys. J. A, 19:283, 2004.

[10] A. Huke, K. Czerski, T. Dorsch, and P. Heide. Evidence for a target-material dependence of the neutron-proton branching ratio in $\mathrm{d}+\mathrm{d}$ reactions for deuteron energies below $20 \mathrm{keV}$. Proceedings of the International Conference on Condensed Matter Nuclear Science, Marseille, France, November 2004. ICCF-11.

[11] K. Czerski, A. Huke, and P. Heide. Electron screening constraints for the cold fusion. Proceedings of the International Conference on Condensed Matter Nuclear Science, Marseille, France, November 2004. ICCF-11.

[12] H. Anderson and J. F. Ziegler. The Stopping and Ranges of Ions in Matter, volume 3. Pergamon Press, New York, 1977.

[13] Ronald E. Brown and Nelson Jarmie. Phys. Rev. C, 41(4):1391, 1990.

[14] H. J. Assenbaum, K. Langanke, and C. Rolfs. Z. Phys., A(327):461-468, 1987.

[15] K. Czerski, A. Huke, P. Heide, and G. Ruprecht. Europhys. Lett., 68(3):363-369, 2004.

[16] S. P. Møller, A. Csete, T. Ichioka, H. Knudsen, U. I. Uggerhøj, and H. H. Andersen. Phys. Rev. Lett., 93:042502, 2004.

[17] William M. Mueller, James P. Blackledge, and George G. Libowitz, editors. Metal Hydrides. Academic Press, New York, London, 1968.

[18] W. Ensinger. Nucl. Instr. Meth., B(127/128):796, 1997.

[19] Andrew Zangwill. Physics at surfaces. Cambridge University Press, Cambridge, 1988.

[20] A. Huke, K. Czerski, and P. Heide. Nucl. Phys. A, 719:279c, 2003.

[21] J. Roth, R. Behrisch, W. Möller, and W. Ottenberger. Nuclear Fusion, 30(3):441, 1990.

[22] A. G. Lipson, A. S. Roussetski, A. B. Karabut, and G. H. Miley. Proceedings of the International Conference on Cold Fusion, Cambridge, MA, USA, 2003. ICCF-10.

[23] E. E. Salpeter. Aust. J. Phys., 7:373, 1954.

[24] T. D. Shoppa, M. Jeng, S. E. Koonin, K. Langanke, and R. Seki. Nucl. Phys., A(605):387, 1996.

[25] M. J. Frisch, G. W. Trucks, et al. Gaussian 94, revision e.1. Technical report, Gaussian Inc., Pittsburgh, 1995. 